\title{
Does the non-union scoring system (NUSS) affect the treatment approach of non-union?
}

\author{
Burcin Karsli ${ }^{1}, \quad$ Sezgin Bahadir Tekin ${ }^{2}, \quad$ Volkan Kılıncoglu1 \\ ${ }^{I}$ Department of Orthopedics and Traumatology, Gaziantep University, Faculty of Medicine, Gaziantep, Turkey \\ ${ }^{2}$ Department of Orthopedics and Traumatology, 25 Arallk State Hospital, Gaziantep, Turkey
}

\begin{abstract}
Aim: To investigate the effectiveness of the non-union scoring system (NUSS) in predicting the result and in guiding the treatment by comparing the treatment methods applied to non-union patients we treat in our clinic with the treatment methods suggested by the NUSS.
\end{abstract}

Methods: The study included 116 patients, who were diagnosed with long bone (femur, tibia and humerus) non-union and treated in our clinic. Of the 116 patients with non-union, 48 had femur (41.38\%), 39 had tibia (33.62\%) and 29 had humerus (25\%) non-union. The patient scores were calculated according to the NUSS criteria. The patients were divided into four groups according to their total scores. There were 34 patients in the first group (0-25 points), 49 patients in the second group (26-50 points), 30 patients in the third group (5175 points) and three patients in the fourth group (76-100 points).

Results: Union that was achieved in $79(68.10 \%)$ of all patients was detected in $97.05 \%$ of the patients in the first group, $83.67 \%$ in the second group, and $16.66 \%$ in the third group. Amputation, arthroplasty and arthrodesis were applied to three patients in the fourth group. While union rate was $100 \%$ in the femur and tibia in the first group, it was $90 \%$ in the humerus. The union rates were $85.71 \%$ in the humerus, $75 \%$ in the femur and $100 \%$ in the tibia in the second group. They were $20 \%$ in the humerus, $15.38 \%$ in the femur and $16.66 \%$ in the tibia in the third group. The number of patients treated with the treatment proposed by the NUSS: $100 \%$ in the group $1,83.67 \%$ in the group $2,20 \%$ in the group 3 and $100 \%$ in the group 4 . The risk of nonunion in those who were not treated according to the NUSS recommendations was 28 times higher than that of others.

Conclusions: The results of our study suggest that more frequent use of the NUSS procedure in non-union treatment planning may increase treatment success. In addition, NUSS can provide information about the treatment process of non-unions.

Key words: Trauma, fracture, pseudoarthrosis, non-union scoring system (NUSS), bone defect.

Dr. Sezgin Bahadir Tekin

Department of Orthopedics and Traumatology, 25 Aralık

State Hospital, Gaziantep, Turkey

E-mail: sezginbahadirtekin@gmail.com

Received: 2021-04-22 / Revisions: 2021-06-24

Accepted: 2021-06-30 / Published online: 2021-10-01

\section{Introduction}

Non-union treatment has always been difficult for orthopedic surgeons. There is no consensus among clinicians, and it was found that there were differences of opinion among clinicians up to $55 \%$ on non-unions [1]. According to the US Food and Drug Administration (FDA), the diagnosis of non-union may be established "after 9 months from injury and if the fracture does not show any progressive signs of healing for 3 months", but others state that for long bones like the femur, humerus or tibia this process can be defined as 6 months if no radiological evidence of fracture healing 
present [2]. On the other hand the treatment of non-union is a challenge and the treatment failure rate of non-union is approximately $\% 20$ [3]. Many classification systems were developed to address this issue. Currently, there are classification systems developed by WeberCech, Ilizarov, and Paley [4, 5, 6]. The most commonly used one is the radiography-based classification system developed by WeberCech. According to this system, non-unions are classified as atrophic, oligotrophic and hypertrophic. While there is a vascular insufficiency in the fracture zone in atrophic and oligotrophic non-unions, vascularity is sufficient in hypertrophic non-union, but reduction is inadequate. It is inevitable that this classification system based on radiography has deficiencies in the treatment-directing.

The new scoring system, Non-Union Scoring System (NUSS), that is described by Calori et al. in 2008, is the most comprehensive classification system ever developed consisting of many parameters [7]. The NUSS is a system in which many factors related to bone, soft tissue and patient are scored, and the treatment options are specified considering the score obtained (Table 1). The NUSS includes the assessment and scoring in terms of the quality of bone, whether the primary injury is open or closed fracture, the number of previous interventions to ensure healing, invasiveness of previous interventions, adequacy of primary surgery, the Weber-Cech classification, bone alignment, bone defect-gap, soft tissues, the ASA grade, diabetes, blood tests (WBC, ESR, CRP), clinical infection status, drugs and smoking status. The score of the patient is calculated by multiplying the sum of the scores by two. In the light of these scores, patients are treated according to the recommended treatment methods in four groups defined. Scores from 0 to 25 is considered straightforward non-unions that are expected to respond well to the appropriate treatments. The problem in this group is generally mechanical. Scores from 26 to 50 would require more specialized care to be given. The problem is mainly biological and mechanical. For patients with scores from 51 to 75 , a specialized care and specialized treatments should be sought. Mechanical and biological failure is more complex and non-union resection and bone defect filling are generally required. Patients with scores over 75 can be candidates for consideration of arthrodesis, arthroplasty or primary amputation [7].

In the present study, the records of patients treated with non-union diagnosis in our clinic were retrospectively reviewed. The results of the treatments were scored according to the NUSS criteria. We investigated the effectiveness of the NUSS in predicting the outcomes and in guiding treatment by comparing the treatment methods applied to non-union patients we treat in our clinic with the treatment methods suggested by the NUSS.

\section{Materials and methods}

The study was retrospectively conducted in our clinic. The study protocol was approved by the local Scientific Research Ethics Committee (2019/04) and the study was conducted in accordance with the Declaration of Helsinki. It included 116 patients treated with long bone (femur, tibia, and humerus) non-union. Inclusion criteria were, the presence of the femur, tibia or humerus non-union, over 18 years of age and the patients with adequate follow-up. Exclusion criteria were, pregnancy, pediatric patients, and fractures due to malignancies and autoimmune diseases. The numbers of male and female patients included in the study are $85(73.3 \%)$ and $31(26.7 \%)$, respectively. The average age of the patients 
Table 1. Non-union scoring system (NUSS).

\begin{tabular}{|c|c|c|c|}
\hline The bone & & Score & $\begin{array}{l}\text { Max. } \\
\text { score }^{\text {a }}\end{array}$ \\
\hline \multirow{4}{*}{ Quality of the bone } & Good & 0 & \\
\hline & Moderate (e.g. mildly osteoporotic) & 1 & \\
\hline & Poor (e.g. severe porosis or bone loss) & 2 & \\
\hline & Very poor (Necrotic, appears avascular or septic) & 3 & 3 \\
\hline \multirow{4}{*}{$\begin{array}{l}\text { Primary injury -open or closed } \\
\text { fracture }\end{array}$} & Closed & 0 & \\
\hline & Open $1^{\circ}$ grade & 1 & \\
\hline & Open $2-3^{\circ}$ A grade & 3 & \\
\hline & Open $3^{\circ} \mathrm{B}-\mathrm{C}$ grade & 5 & 5 \\
\hline \multirow{4}{*}{$\begin{array}{l}\text { Number of previous } \\
\text { interventions } \\
\text { on this bone to } \\
\text { procure healing }\end{array}$} & None & 1 & \\
\hline & $<2$ & 2 & \\
\hline & $<4$ & 3 & \\
\hline & $>4$ & 4 & 4 \\
\hline \multirow{4}{*}{$\begin{array}{l}\text { Invasiveness of previous } \\
\text { interventions }\end{array}$} & Minimally-invasive: Closed surgery (screws, k wires, . . . ) & 0 & \\
\hline & Internal intra-medullary (nailing) & 1 & \\
\hline & Internal extra-medullary & 2 & \\
\hline & Any osteosynthesis which includes bone grafting & 3 & 3 \\
\hline \multirow{2}{*}{ Adequacy of primary surgery } & Inadequate stability & 0 & \\
\hline & Adequate stability & 1 & 1 \\
\hline \multirow{3}{*}{ Weber \& Cech group } & Hypertrophic & 1 & \\
\hline & Oligotrophic & 3 & \\
\hline & Atrophic & 5 & 5 \\
\hline \multirow[t]{2}{*}{ Bone alignment } & Non-anatomic alignment & 0 & \\
\hline & Anatomic alignment & 1 & 1 \\
\hline \multirow{3}{*}{ Bone defect - Gap } & $0.5-1 \mathrm{~cm}$ & 2 & \\
\hline & $1-3 \mathrm{~cm}$ & 3 & \\
\hline & $>3 \mathrm{~cm}$ & 5 & 5 \\
\hline \multicolumn{4}{|l|}{ Soft tissues } \\
\hline \multirow{9}{*}{ Status } & Intact & 0 & \\
\hline & Previous uneventful surgery, minor scarring & 2 & \\
\hline & Previous treatment of soft tissue defect (e.g. skin loss, local flap cover, & 3 & \\
\hline & multiple incisions, compartment syndrome, old sinuses) & \multirow[b]{2}{*}{4} & \\
\hline & Previous complex treatment of soft tissue defect (e.g. free flap) & & \\
\hline & Poor vascularity: absence of distal pulses, poor capillary refill, venous & 5 & \\
\hline & insufficiency & \multirow{3}{*}{6} & \multirow{3}{*}{6} \\
\hline & Presence of actual skin lesion/defect (e.g. ulcer, sinus, exposed bone or & & \\
\hline & plate) & & \\
\hline \multicolumn{4}{|l|}{ The patient } \\
\hline \multirow{2}{*}{ ASA Grade } & 1 or 2 & 0 & \\
\hline & 3 or 4 & 1 & 1 \\
\hline \multirow{3}{*}{ Diabetes } & No & 0 & \\
\hline & Yes - well controlled $(\mathrm{HbA1c}<10)$ & 1 & \\
\hline & Yes - poorly controlled $(\mathrm{HbA} 1 \mathrm{c}>10)$ & 2 & 2 \\
\hline \multirow{3}{*}{ Blood tests: FBC, ESR, CRP } & FBC: WCC $>12$ & 1 & \\
\hline & ESR $>20$ & 1 & \\
\hline & $\mathrm{CRP}>20$ & 1 & 3 \\
\hline \multirow{3}{*}{ Clinical infection status } & Clean & 0 & \\
\hline & Previously infected or suspicion of infection & 1 & \\
\hline & Septic & 4 & 4 \\
\hline \multirow{2}{*}{ Drugs } & Steroids & 1 & \\
\hline & NSAIDs & 1 & 2 \\
\hline \multirow{2}{*}{ Smoking status } & No & 0 & \\
\hline & Yes & 5 & 5 \\
\hline
\end{tabular}

${ }^{a}$ Higher score implies more difficult to procure union. 
Table 2. Number of patients treated regarding treatment proposed by non-union scoring system (NUSS).

\begin{tabular}{|l|c|c|c|}
\hline NUSS Score & $\begin{array}{c}\text { The number of } \\
\text { patients }\end{array}$ & Union & $\begin{array}{c}\text { The number of patients treated regarding } \\
\text { treatment proposed by NUSS/ (\%) }\end{array}$ \\
\hline Group 1 (0-25) & 34 & 33 & $34(\% 100)$ \\
\hline Group 2 (26-50) & 49 & 41 & $41(\% 83,67)$ \\
\hline Group 3 (51-75) & 30 & 5 & $6(\% 20)$ \\
\hline Group 4 (76-100) & 3 & 0 & $3(\% 100)$ \\
\hline Total & 116 & 79 & 84 \\
\hline
\end{tabular}

was 40.5 (17-86). The mean follow-up period of the patients was 21.79 (11-63) months. Pediatric patients were not included in the study. The patients who underwent non-union treatment were retrospectively evaluated according to the Non-Union Scoring System (NUSS) developed by Calori et al. [1]. The number of patients who developed non-union (116 patients) in the femur, tibia, and humerus was $48(41.38 \%), 39(33.62 \%)$ and $29(25 \%)$, respectively. The score of patients was calculated according to the NUSS criteria. The patients were divided into four groups with respect to their total scores. There were 34 patients in the first group (score: 0-25), 49 patients in the second group (score: 26-50), 30 patients in the third group (score: 51-75) and three patients in the fourth group (score: 76100) (Table 2). All patients were followed-up at 1, 3, 6, 9, 12 months by x-rays or CT-scans and union was assessed. Radiological presence of callus formation (3/4 of cortical) in AP-Lateral $\mathrm{X}$-rays and clinical absence of pain in the fracture side were accepted as healing of the fracture.

\section{Statistical analysis}

The normality of distribution of continuous variables was tested by the Shapiro-Wilk test. The Student's $t$ test was used for the comparison of two independent groups of variables with a normal distribution. The relationship between categorical variables was determined by the chi-square test. Univariate logistic regression analysis was used to estimate odds ratio (OR) and $95 \%$ confidence interval. Descriptive statistic parameters were presented as frequency, percentage (\%) and mean \pm standard derivation (mean \pm SD). Statistical analysis was performed with SPSS for Windows version 22.0 and a $p$ value $<0.05$ was accepted to be statistically significant.

\section{Results}

The patients were divided into four groups according to the NUSS score. There were 34 patients in the first, 49 patients in the second, 30 patients in the third and three patients in the fourth group. Union was achieved in 79 $(68.10 \%)$ of all patients. The union rate was determined to be $97 \%$ in the first group, $83.67 \%$ in the second group, and $16.67 \%$ in the third group. On the other hand, union was not achieved in the fourth group. The mean fracture healing time was $6.8 \pm 1.82$ months for the first group, $7.1 \pm 1.55$ months in the second group and $7.82 \pm 1.63$ months in the third group.

Amputation, arthroplasty and arthrodesis were applied to three patients in the fourth group. While union rates were $100 \%$ in the femur and tibia in the first group, it was $90 \%$ in humerus. Union rates in the second group were $85.71 \%$ in humerus, $75 \%$ in the femur and $100 \%$ in the 
Table 3. Our treatment choices for each bone.

\begin{tabular}{|c|c|c|}
\hline Groups & Treatment choices & $\begin{array}{l}\text { The number of } \\
\text { patients }\end{array}$ \\
\hline \multicolumn{3}{|c|}{ Group 1 (NUSS Score 0-25) $(n=34)$} \\
\hline Humerus & Fixation system chanced and autogenous bone grafting & 10 \\
\hline \multirow[t]{2}{*}{ Femur } & Fixation system chanced & 3 \\
\hline & Fixation system changed and autogenous bone grafting & 6 \\
\hline \multirow[t]{2}{*}{ Tibia } & Fixation system chanced & 6 \\
\hline & Fixation system changed and autogenous bone grafting & 9 \\
\hline \multicolumn{3}{|c|}{ Group 2 (NUSS Score 26-50) $(n=49)$} \\
\hline \multirow[t]{2}{*}{ Humerus } & Fixation system chanced & 1 \\
\hline & Fixation system changed and autogenous bone grafting & 13 \\
\hline \multirow[t]{4}{*}{ Femur } & Autogenous bone grafting & 1 \\
\hline & Fixation system chanced & 3 \\
\hline & Fixation system changed and autogenous bone grafting & 18 \\
\hline & Fixation system changed and vascular bone grafting & 2 \\
\hline \multirow[t]{3}{*}{ Tibia } & Fixation system chanced & 3 \\
\hline & Fixation system changed and autogenous bone grafting & 7 \\
\hline & Nonunion resection and segment shifting & 1 \\
\hline \multicolumn{3}{|c|}{ Group 3 (NUSS Score 51-75) $\quad(n=30)$} \\
\hline Humerus & Fixation system changed and autogenous bone grafting & 5 \\
\hline \multirow[t]{3}{*}{ Femur } & Fixation system changed and autogenous bone grafting & 10 \\
\hline & Fixation system changed and vascular bone grafting & 2 \\
\hline & Tumor resection arthroplasty & 1 \\
\hline \multirow[t]{3}{*}{ Tibia } & Fixation system changed and autogenous bone grafting & 8 \\
\hline & Fixation system changed and vascular bone grafting & 1 \\
\hline & Nonunion resection and segment shifting & 3 \\
\hline \multicolumn{3}{|c|}{ 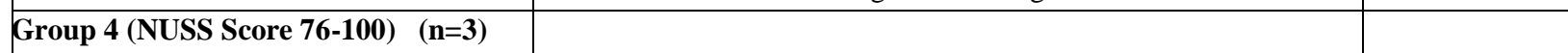 } \\
\hline Humerus & - & \\
\hline \multirow[t]{2}{*}{ Femur } & Arthrodesis & 1 \\
\hline & Tumor resection arthroplasty & 1 \\
\hline Tibia & Amputation & 1 \\
\hline
\end{tabular}

Humerus (n=29); Femur (n=48); Tibia $(n=39)$.

tibia. They were $20 \%$ in the humerus, $15.38 \%$ in the femur and $16.66 \%$ in the tibia in the third group. The fixation system was changed to increase the stability for 34 patients in the first group, and autogenous grafting was performed in addition to the fixation system for 25 patients
(Table 3). The fixation system was changed, and autogenous grafting was performed for 38 of the 49 patients in the second group. Seven patients were treated in this group by changing the fixation system used. Two patients underwent vascular bone grafting. The grafting 
was applied to only one patient. Non-union was resected, and bone defect was treated by segment shifting method in one patient (Table 3). The fixation system was changed, and autogenous grafting was performed for 23 of the 30 patients in the third group. Vascularized bone grafting was performed by changing the fixation system in three patients. Non-union was treated by non-union resection and segment shifting method in three patients. One patient underwent tumor resection arthroplasty (Table 3). The arthrodesis, tumor resection prosthesis, and amputation were applied for each of the three patients in the fourth group separately (Table 3).

The treatment methods applied to the patients were compared with the treatment recommendations proposed by the NUSS (Table 2). While the treatment methods used in the first and fourth groups were completely compatible with those recommended by the NUSS, it was determined that the treatment methods applied to the second and third group are entirely different from those recommended by the NUSS. The success rate in patients treated with the methods proposed by the NUSS was remarkable.

There was a statistically significant relationship between the treatment proposed by the NUSS and union rate $(\mathrm{p}=0.001)$. The risk of non-union in those who were not treated according to the NUSS recommendations was 28 times higher than that of others (Odds Ratio $=28.75 \%$ Confidence Interval =9.66-85.61).

\section{Discussion}

In the present study we evaluated the patients who had developed a non-union. And the NUSS was used for scoring the non-unions and to see the process of fracture healing. There are a few studies in the literature investigating the classification systems for non-union [8-9-10].
We used the NUSS for analyzing the nonunions. There are not many studies about this classification system in the literature. Calori et al. have tested the validity of their classification system in the articles published in 2014 [8] and stated that this system might be a valid guideline. In a retrospective study conducted by Abumunaser et al. [11], 40 patients were divided into three groups, asserting that there would be no clear distinction regarding the treatment protocols between the group 2 (Score: 26-50) and the group 3 (Score: 51-75) in this classification. They stated that their treatment protocols were similar to the treatment protocols described in the NUSS, and reported that they achieved similar success rates in the treatment.

All patients in the first group were treated by the methods the NUSS recommended and union rate achieved was $97.05 \%$. In the second group, $83.67 \%$ of the treatments were compatible with the methods recommended by the NUSS, and the union rate was $83.67 \%$. On the other hand, only $20 \%$ of treatment methods applied to the patients in the third group complied with the treatment recommendations of the NUSS. The treatment success rate in this group was only $16.67 \%$. Those results revealed that the non-union risk was 28 times higher in the patients who were not treated using the methods recommended by the NUSS when compared to patients treated according to the NUSS recommendations. This results were similar the study that published by Calori et al. [8]. In their study they analyzed 300 patients that applied with long bone non-unions.

According to the NUSS, the main problem in the first group is mechanical, and the aim of the treatment is to change the fixation system. In the present study, the fixation system was changed to improve stability in all patients in the first group. Treatment success was achieved 
when the mechanical problem was solved. The problem in the second group was generally biological and mechanical. Recovery of fixation and the provision of biological stimulant are suggested for treatment. A more stable fixation was provided by changing the fixation system in $83.67 \%$ of the patients we treated in this group and biological support was given with an autogenous iliac bone graft. Union was achieved in these patients. In this group, only the fixation system was changed in seven out of eight patients whose union was not achieved with the treatment, and no biological stimulation was used. Autogenous bone grafting was performed in a patient whose fixation system was not changed. According to the NUSS, the problem is complex in the third group and there is an impairment of both biological and mechanical conditions. Nonunion should be resected as the treatment suggestion and the bone defect should be treated. In our series, the treatment protocol suggested by the NUSS was applied only in six patients in this group, and union was achieved in five patients. Thus, the success rate was $83.33 \%$ in the treatment. However, union was not achieved in 25 patients in the group. Low success rate in this group may be related to our failure to use more aggressive treatment protocols. The treatment we applied was mostly changing the fixation system and autogenous grafting. Application of more effective methods like segment shifting and vascular grafting after non-union resection could have increased our treatment like our study [12].

The NUSS recommends treatments such as arthrodesis, arthroplasty and amputation for the fourth group. We applied tumor resection arthroplasty, arthrodesis and amputation for our patients in this group.

There are several limitations in the study. The limitations of the study are that the NUSS has yet to be validated [8] and the study was conducted retrospectively. However, it can be thought that as the number of studies on this subject increase, the scoring system will be used more widely.

We think that the more frequent use of the NUSS in non-union treatment planning can increase the success of the treatment and it can be used as a classification guide for the surgeon in the treatment of nonunion.

Funding: The author(s) received no financial support for the research, authorship, and/or publication of this article.

Conflict of Interest: The authors declare that they have no conflict of interest.

\section{Ethical statement:}

The study was approved by Local Clinical Research Ethics Committee (Date and Decision Number: 2019/04) and written informed consent was obtained from each subject.

\section{Open Access Statement}

This is an open access journal which means that all content is freely available without charge to the user or his/her institution under the terms of the Creative Commons Attribution NonCommercial License (http://creativecommons.org/licenses/bync/4.0). Users are allowed to read, download, copy, distribute, print, search, or link to the full texts of the articles, without asking prior permission from the publisher or the author.

Copyright (c) 2021: Author (s).

\section{References}

[1]Bhandari M, Fong K, Sprague S, et al. Variability in the definition and perceived causes of delayed unions and nonunions: a cross-sectional, multinational survey of orthopaedic surgeons. J Bone Joint Surg Am. 2012;94(15):e1091-96. 
[2]Fayaz HC, Giannoudis PV, Vrahas MS, et al. The role of stem cells in fracture healing and nonunion. Int Orthop. 2011;35(11):1587-97.

[3]Tzioupis C, Giannoudis PV. Prevalence of long-bone non-unions. Injury. 2007;38 Suppl 2:S3-9.

[4]Weber BG, Cech O. Pseudarthrosis. Grune and Stratton, New York; 1976.

[5]Catagni MA. Treatment of fractures, nonunions, and bone loss of the tibia with the Ilizarov method. Ed Maiocchi AB;1998. 7:90.

[6]Paley D, Catagni MA, Argnani F, et al. Ilizarov treatment of tibial nonunions with bone loss. Clin Orthop Relat Res. 1989;(241):146-65.

[7]Calori GM, Phillips M, Jeetle S, Tagliabue L, Giannoudis PV. Classification of nonunion: need for a new scoring system? Injury. 2008;39 Suppl 2:S59-63.

[8]Calori GM, Colombo M, Mazza EL, et al. Validation of the Non-Union Scoring System in 300 long bone non-unions. Injury. 2014;45 Suppl 6:S93-97.

[9]Megas P. Classification of non-union. Injury. 2005;36 Suppl 4:S30-7.

[10]Frölke JP, Patka P. Definition and classification of fracture non-unions. Injury. 2007;38 Suppl 2:S19-22.

[11] Abumunaser LA, Al-Sayyad MJ. Evaluation of the calori et $\mathrm{Al}$ nonunion scoring system in a retrospective case series. Orthopedics. 2011;34(5):359.

[12] van Basten Batenburg M, Houben IB, Blokhuis TJ. The Non-Union Scoring System: an interobserver reliability study. Eur J Trauma Emerg Surg. 2019;45(1):1319. 